\title{
PR-9. CYANMORPHOLINOETHYLENE IN THE SYNTHESIS OF RELEVANT AZOLOAZINES
}

\author{
V. V. Fedotov, K. V. Savateev, M. A. Maltsev, E. N. Ulomsky, V. L. Rusinov \\ Ural Federal University of the first President of Russia B. N. Yeltsin, \\ Mira St., 19, Yekaterinburg, 620002, Russia \\ E-mail: vicww4@gmail.com
}

Heterocycles are impressively presented as components of many drugs with different types of biological activity, such as antimicrobial, anticancer, antiviral. Azolopyrimidines are practically important group of heterocyclic compounds due to their useful biological effects. Based on the literature search, it was found that primary amino fragment makes a significant contribution to the affinity towards adenosine receptors, and interaction with this type of receptors is a possible mechanism for the formation of antiseptic action of azoloazine compounds. Considering this it's actual to develop synthetic methods of azoloazines containing primary amino fragment.

Cyanmorpholinoethylene was obtained by the method described in the literature, which consists in triple condensation of cyanacetic acid with the appropriate amine and triethylorthoformate. The use of morpholine as a component of this condensation was considered to be the most preferable, as it allows to obtain a product with a higher yield $(85 \%)$ in comparison with piperidine $(60 \%)$ or diethylamine $(55 \%)$.

The further interaction of the obtained cyanmorpholinoethylene with variously substituted aminoazoles was performed according to scheme.

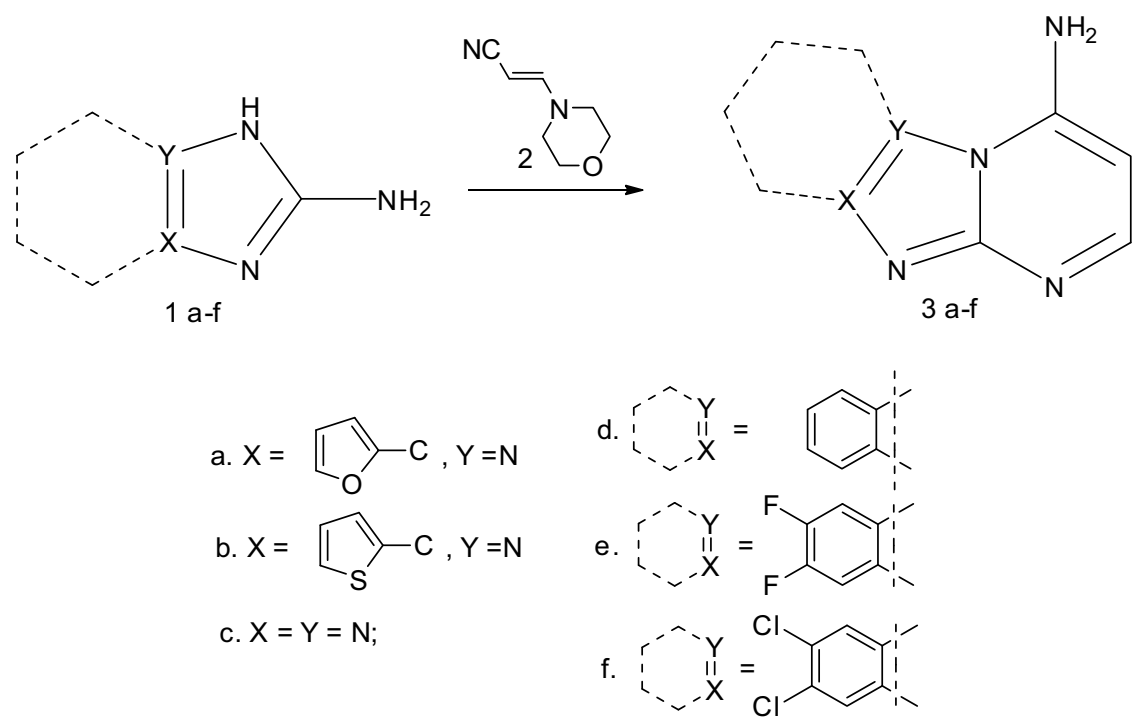

Thus, the result of the research is design and optimization of the approach to the synthesis of promising azolopyrimidines containing a primary amino group by cyclocondensation of available aminoazoles and cyanmorpholinoethylene in equimolar mixture of pyridine and acetic acid or piridine itself as the reaction medium.

The results were obtained within the framework of the state task of the Ministry of education and science of Russia (4.6351.2017/8.9) and with the financial support of the Russian science Foundation (№ 17-13-01096). 